\title{
Protein quantification by LC-MS: a decade of progress through the pages of Bioanalysis
}

\author{
Nico C van de Merbel*,1,2 \\ ${ }^{1}$ Bioanalytical Laboratory, PRA Health Sciences, Amerikaweg 18, 9407 TK, Assen, The Netherlands \\ ${ }^{2}$ Department of Analytical Biochemistry, Groningen Research Institute of Pharmacy, University of Groningen, Antonius Deusinglaan \\ 1, 9713 AV, Groningen, The Netherlands \\ *Author for correspondence: MerbelNicovande@prahs.com
}

Over the past 10 years, there has been a remarkable increase in the use of LC-MS for the quantitative determination of proteins, and this technique can now be considered an established bioanalytical platform for the quantification of macromolecular drugs and biomarkers, next to the traditional ligand-binding assays. Many researchers have contributed to the field and helped improve both the technical possibilities of LC-MS-based workflows and our understanding of the meaning of the results that are obtained. As a tribute to Bioanalysis, which has published many important contributions, this report gives a high-level overview of the most important trends in the field of protein LC-MS, as published in this journal since its inauguration a decade ago. It describes the major technical developments with regard to sample handling, separation and MS detection of both digested and intact protein analysis. In addition, the relevance of the complex structure and in vivo behavior of proteins is discussed and the effect of protein-protein interactions, biotransformation and the occurrence of isoforms on the analytical result is addressed.

First draft submitted: 12 February 2019; Accepted for publication: 12 March 2019; Published online: 15 April 2019

Keywords: biomarkers $\bullet$ biopharmaceuticals $\bullet$ biotransformation $\bullet$ digestion $\bullet$ HRMS $\bullet$ immunocapture $\bullet$ isoforms - LC-MS • proteins

Over the past decade, since the inception of this journal, there have been many developments in the field of bioanalysis, but one of the most prominent probably is the rise of LC-MS for the quantification of proteins. While the determination of macromolecular biomarkers and biopharmaceuticals typically was the realm of ligand-binding assays (LBAs) 10 years ago, with LC-MS mainly being used for small molecules, it is fair to say that LC-MS has now also become an established platform for protein quantification. Because of its fundamentally different analytical principles, LC-MS can provide qualitative and quantitative information about proteins in biological samples that is complementary to LBA results. As such, it can contribute significantly to understanding the in vivo fate of dosed or endogenous macromolecules, whose structure and properties are so much more complex than those of traditional drugs and biomarkers of low molecular weight. In some cases, LC-MS may even be the only technique capable of providing a useful quantitative result.

Since the first quantitative bioanalytical LC-MS methods for proteins started to be reported, a little over 10 years ago, many scientists have contributed to the development of the field. LC-MS-based bioanalysis for proteins is different in many ways from small-molecule LC-MS as well as from LBAs, so much needed to be learned. Understandably, developments started with a clear focus on the technological aspects and much effort has gone into optimizing and properly combining different analytical tools to allow quantification of proteins with the required sensitivity, selectivity, accuracy and precision. While with a well-developed LC-MS assay for a small molecule, there generally is no discussion about what a concentration result represents, it was soon realized that this is not so straightforward for large and structurally complex proteins. LC-MS, like most LBAs, usually targets only a part of the analyte, and the concentration result obtained is strongly dependent on the exact part of the protein to which the method is directed and on the underlying analytical principle of the assay. Consequently, the field of protein bioanalysis has seen increasing attention for what a concentration result, generated by LC-MS, exactly represents. 


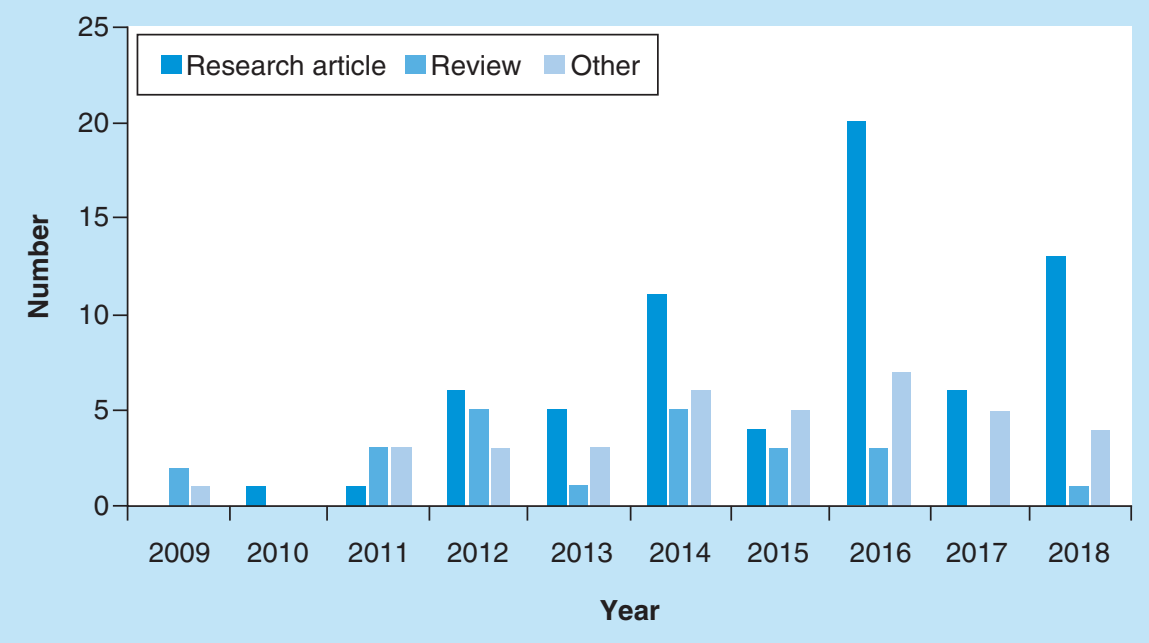

Figure 1. Historical overview of research papers, reviews and other contributions (editorials, perspectives, commentaries) on protein LC-MS, as published in Bioanalysis since 2009.

Many important contributions in the area of LC-MS-based protein quantification have been published in Bioanalysis (Figure 1). As a tribute to the 10-year anniversary of the journal, this report gives a high-level overview of the most important developments and trends in the field, which have appeared on the pages of Bioanalysis in the past decade. This overview is by no means meant as an exhaustive review of the literature, because many key papers have obviously also appeared in other journals. Among the many papers published in this journal, there are several excellent reviews of different aspects of this broad field, to which the interested reader is referred for more detailed information.

\section{The analysis}

\section{Digested protein analysis}

The traditional approach of protein quantification by LC-MS was, and often still is, the enzymatic digestion of a protein analyte into a mixture of peptides and the quantification of one or a few of these as a surrogate readout for the intact protein. The advantages are obvious. Large proteins are typically difficult to quantify by the classical combination of a reversed-phase LC column and a triple-quadrupole mass spectrometer. Enzymatic digestion reduces the complicated analyte into a much more manageable set of smaller peptides, and their quantification can be based on the decades of accumulated knowledge that we have of small-molecule and, especially, peptide LC-MS. At the same time, protein quantification can readily benefit from the general technical advantages of LC-MS, such as a wide linear dynamic range, good accuracy and precision because of the ability to apply internal standards, the possibility of quantifying multiple analytes simultaneously and the independence of critical immunoreagents of potentially poor quality.

\section{Digestion}

Not surprisingly, there was and is much enthusiasm in the bioanalytical community, and notably among LC-MS scientists, about the potential of this technique for protein quantification. Several review papers were published in this journal on the technical aspects of targeted LC-MS quantification of proteins as early as 2011 [1-3]. They emphasized the importance of the digestion step as the central part of the workflow, and the selection of a proper surrogate peptide for quantification, but otherwise approached protein bioanalysis essentially as an extension of peptide bioanalysis. Developments have been rather limited, when it comes to the digestion step. By far most researchers are using trypsin as the digestion enzyme of first choice [4]. This is mainly due to its wide availability for a reasonable price and its ability to cleave proteins into peptides of a size that is compatible with LC-MS/MS analysis on a triple quadrupole. If no suitable peptides are formed by trypsin, alternative enzymes such as chymotrypsin [5] or Glu-C [6] can also be used, or even chemical digestion, such as in the case of the presence of an acid-labile amide bond in the protein of interest [7]. 
Plasma or serum can be directly digested in solution [1-3], but it is very useful to first isolate the protein fraction from the sample by protein precipitation and centrifugation, and then resuspend and digest the obtained protein pellet. This approach, known as pellet digestion [8,9], has the advantage that it concentrates the sample and that some matrix interferences, such as smaller proteins and phospholipids, are removed. Traditionally, to ensure its completeness, digestion is often performed overnight, which makes protein quantification a 2-day process. Although undesirable from a sample throughput perspective, it is an approach that is still used in many recent reports, even though digestion times can be reduced to a few hours, if properly optimized, and even to a matter of minutes when using an immobilized trypsin reactor $[10,11]$. An interesting innovation worth mentioning is the concept of limited proteolysis, which was reported for the quantification of a monoclonal antibody (mAb) [12]. In this approach, the protein analyte is selectively captured within the relatively small pores of a resin, while trypsin is immobilized on a larger particle, which cannot completely penetrate into the pore. In this way, only a small part of the analyte is digested, which reduces the complexity of the digest.

Most published digestion protocols include denaturation, reduction and alkylation steps, prior to digestion, to unfold the protein structure and break intramolecular disulfide bonds. This typically makes the inner part of a protein more accessible to the digestion enzyme and will speed up the digestion process, but these steps do not necessarily improve the overall analysis. If the surrogate peptide is located at the protein surface and does not contain a disulfide-forming cysteine it may be beneficial to skip those steps, to reduce the formation of interfering peptides originating from endogenous proteins [13].

\section{Protein \& peptide enrichment}

Despite all advantages, it was also evident that LC-MS suffers from a major technological drawback compared with an LBA: its relatively limited sensitivity, and much scientific effort has gone into addressing and improving this situation. An important reason for the limited sensitivity of LC-MS for protein bioanalysis is the high abundance of endogenous proteins in complex matrices such as serum and plasma. If these proteins are all also digested, an even more complex mixture is formed, containing of a myriad of peptides with very similar analytical properties. Many of these peptides have the same or nearly the same mass, and the same fragmentation behavior, as the surrogate peptide and show up as interferences in the chromatograms. In this way, the matrix background can be responsible for a reduction of more than a factor 100 in the achievable LLOQ [14]. Consequently, for matrices with a lower endogenous protein content, such as bronchoalveolar lavage fluid, obtaining a good sensitivity is usually much easier [15].

The enrichment of the protein analytes from the biological matrix and/or the surrogate peptides from the digest is an obvious way to improve sensitivity and much research has been devoted to this over the years [16]. As reviewed in 2015 [17], a variety of generic enrichment methods have been developed, most successfully if the protein analyte is structurally different from the bulk of the endogenous matrix proteins. PEGylated proteins, for example, can easily be enriched by extraction into an organic solvent. Solid-phase extraction on ion-exchange materials is a possibility if a protein's [18] or peptide's [19] isoelectric point is clearly different from that of their endogenous counterparts, while molecules containing histidine moieties on their surface can be isolated by complexation with immobilized metal ions, such as nickel, an approach called immobilized metal affinity chromatography [20,21]. By applying one or a combination of these techniques, protein quantification in complex biological matrices down to low- to sub-ng/ml level has been achieved, but a good knowledge of the analyte's properties is essential and there is no guarantee that the required sensitivity can indeed be reached.

\section{Affinity capture}

If more selectivity and sensitivity are needed, enrichment based on analyte-specific affinity interaction often is the better option [22,23]. It should be kept in mind, however, that the reagents needed for affinity capture in principle suffer from the same disadvantages as those used for LBAs, such as batch-to-batch differences and limited availability, which could remove one of the advantages of using LC-MS over LBA. Still, many researchers appear to have ready access to reagents of sufficient quality and several published LC-MS methods for proteins include an affinity capture step. Affinity-based enrichment can also be quite generic, such as the use of protein A or protein $\mathrm{G}$ to capture therapeutic antibodies of the IgG type from a biological sample [24], although it is a disadvantage that the very abundant and heterogeneous endogenous IgG fraction in the plasma of many species also binds to protein A/G. A more selective alternative is affinity capture using an antibody directed to the constant $(\mathrm{Fc})$ part of human IgG to selectively isolate a variety of human or humanized mAbs from animal matrices [25,26]. Like in 


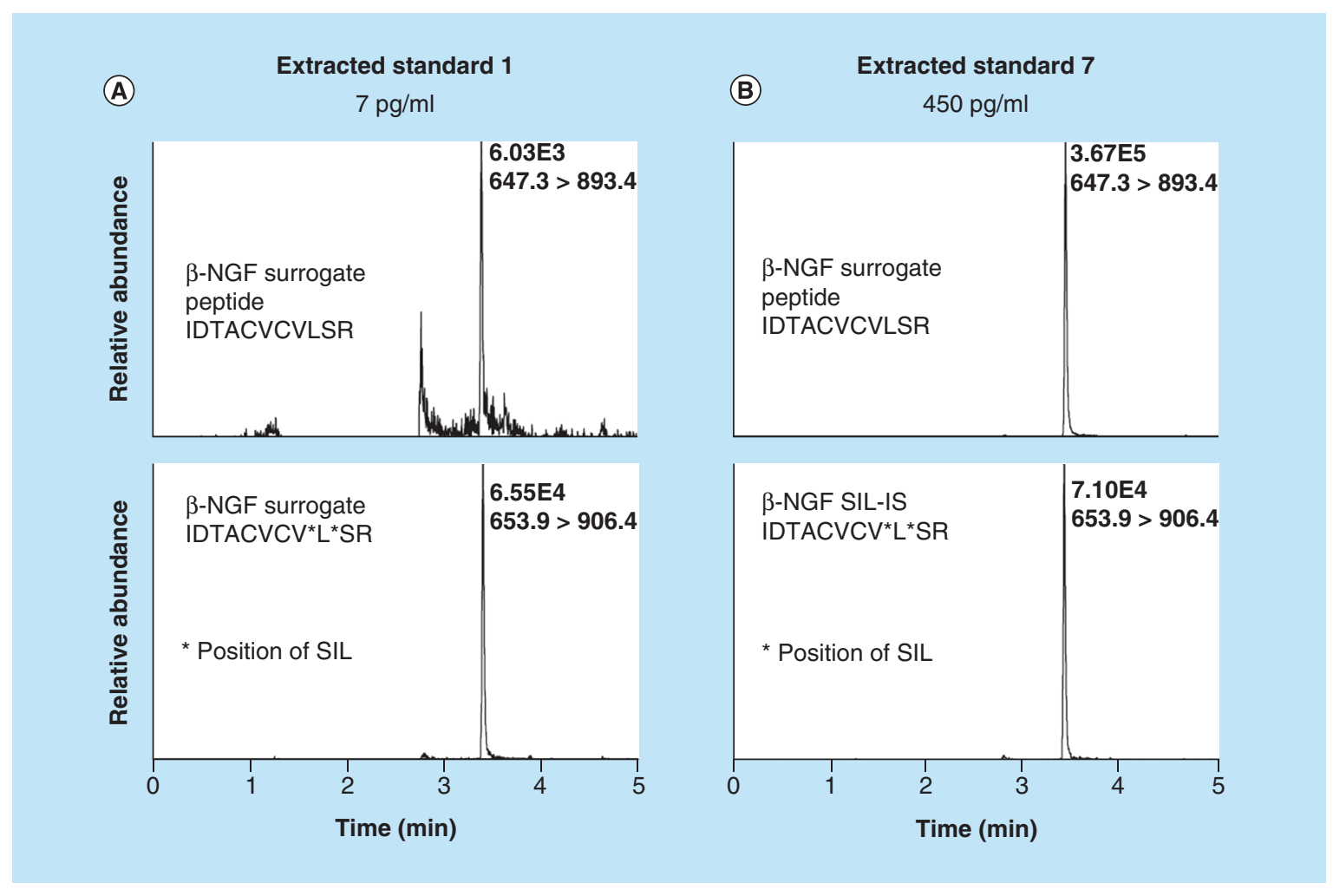

Figure 2. LC-MS/MS chromatograms of the surrogate peptide and the corresponding internal standard of $\beta$-nerve growth factor. LLOQ standard at $7 \mathrm{pg} / \mathrm{ml}$ (A) and ULOQ standard at $450 \mathrm{pg} / \mathrm{ml}$. Protein extracted from plasma and peptide extracted from digest by immunocapture. Reproduced from [36] (c) Future Science Group (2016).

SIL: Stable-isotope labeled.

LBAs, ultimate selectivity is obtained with a capturing reagent that is specifically directed to the analyte of interest, for example, an anti-idiotypic antibody [27] or the pharmacological target [28]. In this way, sensitivity down to the $\mathrm{pg} / \mathrm{ml}$ level can typically be reached.

Recently, alternative materials have emerged for affinity capture. Aptamers, for example, are oligonucleotide molecules [29] and affimers are engineered polypeptides [30], that both bind to proteins with high affinity and have been used for quantitative bioanalysis of proteins. In addition, synthetic molecular imprinted polymers (MIPs) can also extract proteins from biological samples and offer a further diversification of affinity capture approaches [31]. While most researchers use reagents immobilized on magnetic beads, which are mixed with the sample and subsequently collected by applying a magnet, other formats have also been developed and successfully used. Examples include the immobilization of a capturing agent in the wells of an ELISA plate [32] and on a monolithic material present in a pipette tip, which aspirates and dispenses the sample multiple times [33]. Affinity capture is usually performed on the intact protein analyte before digestion but can equally well be done after digestion. In that case, a capture reagent is used which selectively extracts the surrogate peptide(s) from the digest [34,35]. In case extreme sensitivity is needed, both pre- and postdigestion affinity enrichment can be combined, as was convincingly demonstrated for the biomarker $\beta$-NGF (Figure 2). By capturing the protein with a polyclonal antibody immobilized on magnetic beads, digesting it with trypsin and subsequently capturing the surrogate peptide on a column containing an antipeptide antibody, concentrations down to $7 \mathrm{pg} / \mathrm{ml}$ could be routinely quantified [36].

\section{Liquid chromatography}

After digestion of a protein analyte and further purification of the sample, the actual analysis typically is not much different from standard small-molecule quantification. Reversed-phase LC in combination with positive-mode electrospray tandem mass spectrometry is the gold standard. Run times tend to be somewhat longer than for small 
molecules, especially if the digest has a complex composition and separation of the surrogate peptide from interfering matrix peptides is needed. Ultra-high-performance LC columns with sub-2 $\mu \mathrm{m}$ particles are increasingly being used to decrease analysis time and/or improve resolution [37]. If sensitivity is not limited by matrix interferences, lower detection limits can generally be obtained by analyzing the same volume of processed sample on a column with a reduced diameter and a lower mobile-phase flow rate [38]. Technical advances in this field of micro- or nano-LC were reviewed in 2014 and it was concluded that the benefits are most effectively realized in combination with a thorough sample cleanup such as immunodepletion and immunoaffinity enrichment [39]. The use of special equipment with extremely low dead volumes is essential, but robust commercial systems are now widely available. The high separation power of LC has led some researchers to use this technique as a sample preparation tool. By collecting the mobile-phase fraction containing the surrogate peptide and injecting it into a second LC system with complementary selectivity, additional cleanup can be achieved, albeit at the expense of a limited sample throughput $[40,41]$. Opportunities for coupling these two LC steps on-line in a 2-D system are there [42] but have certainly not been applied on a large scale.

Without doubt, the possibility to determine several compounds in a single run is a very attractive feature of LCMS in comparison with the typical LBA, although the majority of published methods is still restricted to a single analyte. The simultaneous quantification of two biopharmaceuticals in plasma, given as a combination therapy [43] or dosed together for research purposes [18], has been reported, as well as the measurement of a biopharmaceutical protein together with its pharmacological (soluble) target [44]. The LC-MS quantification of two appropriate surrogate peptides for such a combo-assay is relatively straightforward, but special attention might be needed to simultaneously extract the two protein analytes from the matrix, for example, by two different affinity capture reagents.

\section{Mass spectrometry}

Surrogate peptides can robustly be quantified on our bioanalytical workhorse, the triple-quadrupole mass spectrometer, by detection of a peptide fragment after collision-induced fragmentation in the multiple reaction monitoring (MRM) mode. Depending on its size and structure, a peptide may form ions with several charge states, but one of these usually dominates in terms of intensity and is, thus, selected for quantification. If needed, the responses of multiple peptide ions can be summated for improved sensitivity.

A recent development is the introduction of high-resolution mass spectrometry (HRMS) for quantitative bioanalysis, usually in the form a quadrupole-time of flight (Q-TOF) instrument. The main advantage of HRMS for the quantification of surrogate peptides in a complex digest is its improved ability to separately detect the responses of molecules with very close molecular masses, and therefore provide a more selective detection of a surrogate peptide. By narrowing the mass extraction window from $0.7 \mathrm{Da}$, the typical value for a triple quadrupole, down to, for example, $0.01 \mathrm{Da}$, most of the ions of a surrogate peptide of interest can still be detected, but a major part of the interferences from digested plasma proteins is no longer selected for detection. This better selectivity may, however, not always lead to better quantification limits, because the absolute instrument sensitivity of triple-quadrupole MS still is superior to that of HRMS, although the difference has decreased over the years. In a report published in 2012, LC-MS/MS quantification of a surrogate peptide on a Q-TOF MS was ten-times less sensitive than on a triple quadrupole [45], but a publication from 2018 showed a fourfold improvement of the achievable LLOQ on a Q-TOF compared with triple-quadrupole MS, even though the absolute instrument sensitivity of HRMS still was less favorable [46]. Analysis on a Q-TOF in the MRM mode (unit mass resolution in the quadrupole followed by high resolution in the TOF) is essential for a good signal to noise ratio: the LLOQ for an mAb in plasma obtained using TOF only without fragmentation of the surrogate peptide was found to be 25-times higher than for Q-TOF analysis in MRM mode [47].

An interesting and relatively unexplored area is the application of supercharging reagents to improve the sensitivity of MS detection of peptides. By adding a specific chemical such as m-nitrobenzyl alcohol to the LC mobile phase, the ionization process of a surrogate peptide (and other peptides) is changed. Generally, the absolute ionization efficiency is increased as well as the relative intensity of the more highly charged species, but background noise may also increase [48]. This approach has the potential to contribute to the improvement of the sensitivity of protein bioanalysis, although more research is clearly needed to establish its full potential. 


\section{Note}

An attractive feature of digested protein analysis is the possibility to use a single peptide as a surrogate for multiple proteins. Rather than using a surrogate peptide from the variable region of an $\mathrm{mAb}$, a so-called universal peptide is selected from the constant region of human IgG. Such a peptide is present in the sequence of all human therapeutic $\mathrm{mAb}$ (candidate) drugs but is not found in the endogenous proteins of any animal species. Thus, a single analytical method can be used to support the preclinical development of a diversity of human mAbs across multiple species, which eliminates the need for repeated separate method development [49]. Methods for seven different mAbs in serum of three different nonhuman species were set up and successfully validated in this way [50]. The approach can, unfortunately, not be used for clinical bioanalysis, because the amino acid sequence of any possible universal peptide will always occur in endogenous human IgG. The only exception was shown for a therapeutic mAb of the IgG4 subclass, which had been structurally modified to stabilize the hinge region and which contains a peptide with a sequence that does not occur in endogenous human antibodies [51].

\section{Intact protein analysis}

Over the past few years, reports have started to appear on the quantification of intact proteins by LC-MS. Despite the success of digestion-based methods, there is an increasing awareness that a surrogate peptide may represent no more than a few percent of the total protein and that, even if multiple peptides are monitored, information about most of the protein structure is lost. In an early review paper of 2015 about the quantification of intact proteins [52], a summary of the existing literature was provided. At the time, nearly all published methods were for small proteins with molecular masses below $15 \mathrm{kDa}$, but more recently much larger intact proteins have also been successfully quantified.

\section{Mass spectrometry}

While triple-quadrupole MS systems have been primarily used for surrogate peptide quantification after protein digestion, intact proteins typically need HRMS. Although the orbitrap platform provides the best mass resolution and has been applied for intact protein quantification [53], most quantitative LC-MS methods published in Bioanalysis use Q-TOF instrumentation. Sensitivity easily becomes an issue with increasing molecular weight, because of the distribution of the detection signal over more and more charge states, each with their own isotope distribution. The 14-kDa test protein lysozyme, for example, showed six different charge states and the summation of the most abundant isotope peak of each charge state was needed for detection down to the $\mathrm{ng} / \mathrm{ml}$ range [54]. The mass spectrum of large proteins, such as mAbs $(150 \mathrm{kDa})$, can be deconvoluted using dedicated software and all (typically about 30) charge states are converted into the corresponding and much simplified neutral spectrum, which is subsequently used as the basis for quantitation (Figure 3). The proof of principle of this approach was demonstrated for the determination of a $\mathrm{mAb}$ in plasma [55]. The summation of the three most abundant charge states of this protein showed similar selectivity, accuracy and precision as the deconvolution approach and allowed quantification down to $50 \mathrm{ng} / \mathrm{ml}$ in plasma [56]. For another $\mathrm{mAb}$, the summation of responses of up to 18 different charge states resulted in better data for precision and accuracy than deconvolution [56], while for an intact antibody-drug conjugate (ADC), deconvolution gave equally acceptable results as quantification based on the response of a single-charge state [57]. For a number of $\mathrm{mAb}$ subunits $(12-25 \mathrm{kDa})$, it was shown that the use of a limited number of charge states provided the best signal-to-noise ratio and that deconvolution can be comparable to charge state quantification but may also be worse [58].

The traditional LC-MS quantification of an intact protein occurs under denaturing conditions, which means that its $3 \mathrm{D}$ conformation is typically lost. Replacing settings like high organic solvent concentrations and low $\mathrm{pH}$ values by milder conditions allows analysis of proteins in their native state, which could be more closely related to their active form. The feasibility of quantification by native LC-MS was demonstrated for a mAb [59]. Considerably fewer charge states were observed, and the achieved sensitivity of $5 \mu \mathrm{g} / \mathrm{ml}$ was 25 -fold less favorable than under denaturing conditions due to the suboptimal electrospray solvents, but the method was successfully validated and applied to preclinical samples.

\section{Liquid chromatography}

Developments in the chromatographic separation of intact protein species have been relatively slow over the past years. Typically, stationary phases with large pores are used at high temperatures to minimize band broadening due to slow mass transfer. Reversed-phase LC on columns packed with C4 phases with 300-1000 $\AA$ pores have 


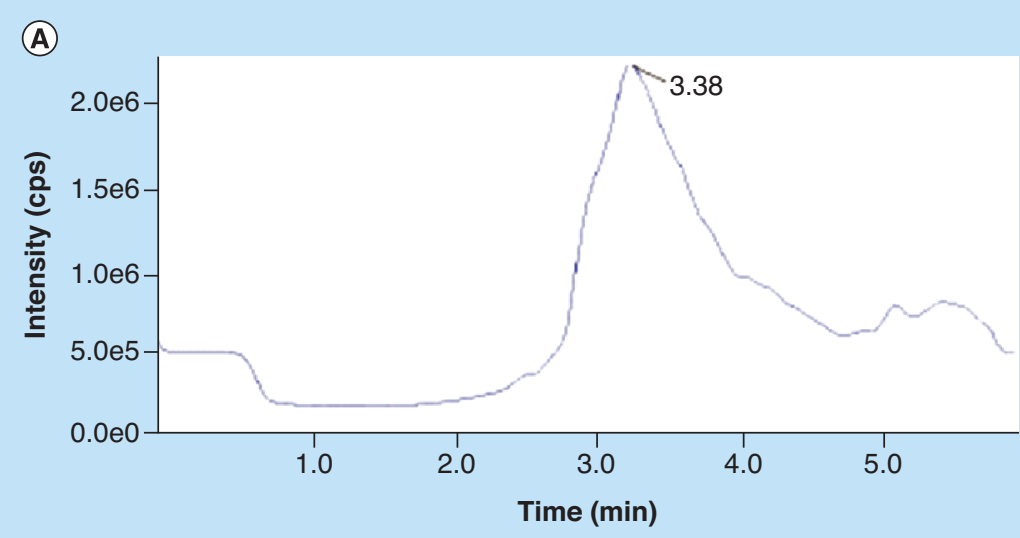

(B)
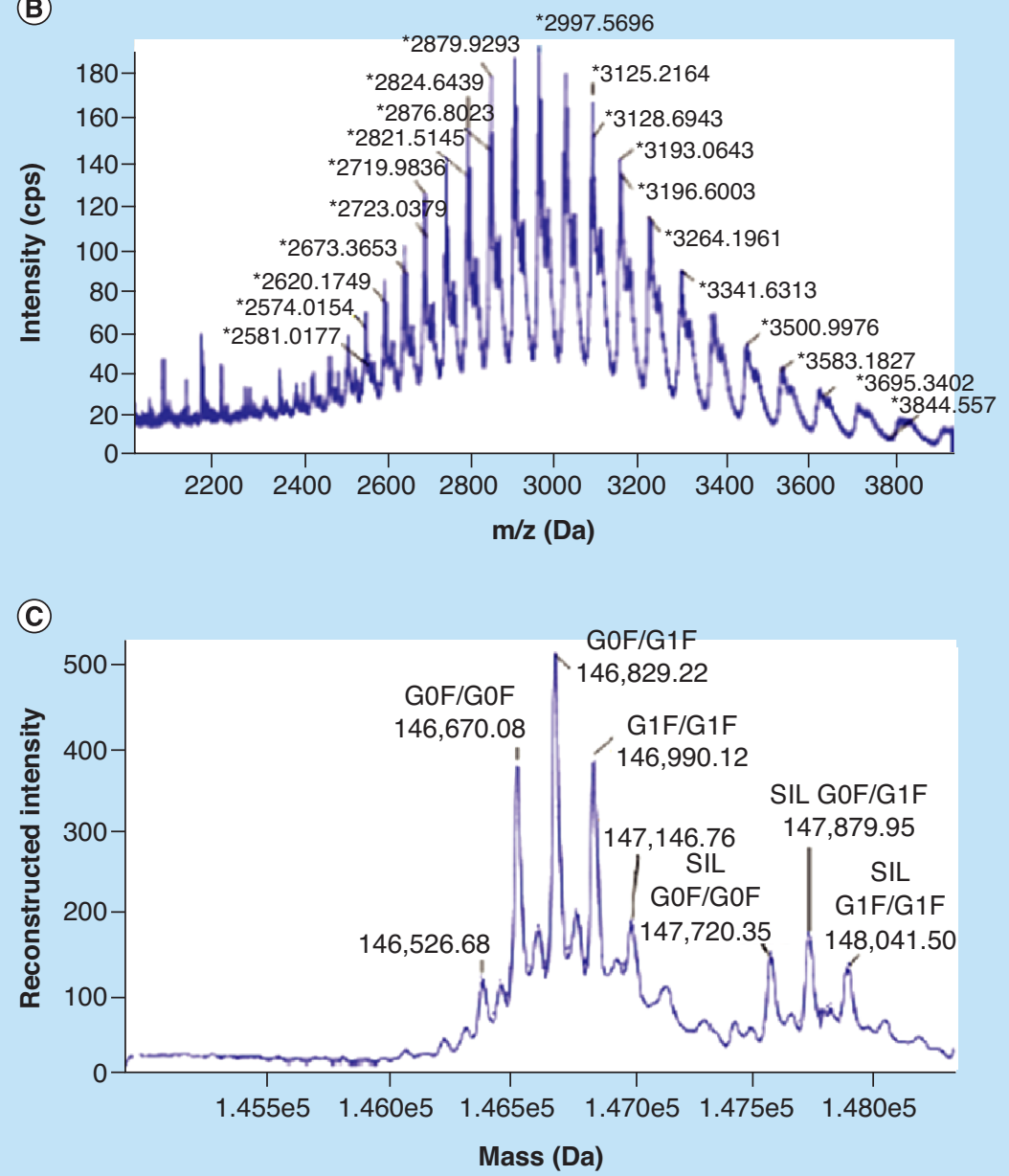

Figure 3. LC-HRMS analysis of a monoclonal antibody at $15 \mu \mathrm{g} / \mathrm{ml}$ in mouse plasma. (A) Chromatogram of a full TOF scan; (B) Raw mass spectrum for the peak at $3.38 \mathrm{~min}$; (C) Deconvoluted mass spectrum showing multiple glycoforms (GOF and G1F) of analyte and SIL internal standard.

SIL: Stable-isotope labeled; TOF: Time of flight.

Reproduced from [55] (c) Future Science Group (2016). 
been most widely used, in combination with a water/acetonitrile gradient in the presence of $0.1 \%$ formic acid and at a temperature of typically $70^{\circ} \mathrm{C}$. Examples of bioanalytical methods for intact mAbs and ADCs on both conventional bore $[54,55,57]$ and microbore LC [60] have been published. Alternatively, monolithic LC columns can be used for protein separation. A monolith is a single polymer rod, which contains a network of through pores and has improved mass transfer properties. As such, it is attractive for protein separation, which is inherently hampered by slow diffusion rates. A 1-mm i.d. monolithic polymer LC column was used under reversed-phase conditions in a method for the quantification of an intact mAb in plasma [56]. Native LC-MS obviously needs chromatographic conditions that keep the protein structure in its natural conformation, so reversed-phase LC with its high concentrations of organic modifiers is not ideal. A wholly aqueous, 2D LC system, in which size-exclusion and weak cation-exchange chromatography were coupled, has been described for the quantification of an mAb in plasma under native conditions [59].

\section{Protein enrichment}

LC-MS of large intact proteins needs a more thorough sample cleanup than LC-MS/MS of digested proteins, mainly because large endogenous proteins will also produce highly complex MS that will easily interfere with the analyte ions used for quantitation. Therefore, a very selective extraction of the protein analyte is essential. All published methods for quantification of an intact mAb by LC-MS include an immunocapture step, either using an antihuman IgG antibody (for analysis of animal samples) [54-57] or an anti-idiotypic antibody (for analysis of human samples) [60]. For native LC-MS, elution of the protein from the capture material has to take place under nondenaturing conditions, such as by an acetate buffer at $\mathrm{pH} 4$ [59]. Cleavage of an $\mathrm{mAb}$ into 25-kDa fragments, by digestion with the enzyme IdeS followed by disulfide reduction, opens up the possibility to reduce the size of protein species and simplify their analysis. In this way, the glycosylation pattern of an mAb can be studied by LC-HRMS via the deconvoluted mass spectra of the different glycoforms [60].

\section{The result}

Because of their structural complexity, quantitative bioanalysis for proteins is not as straightforward as for small molecules. Usually, only a specific part of a protein molecule responds to an analytical technique and, therefore, different concentration results may be obtained for a given sample via different techniques, depending on their analytical principle and the part of the protein to which they are directed. For a meaningful concentration result, it is not sufficient to analyze samples with proper sensitivity, selectivity, precision and accuracy, but it is also important to understand what exactly the result represents [61]. Proteins often have different isoforms (including a variety of post-translational modifications), they can occur in vivo in free and bound form and may undergo biotransformation during their residence in the body (Figure 4) [62], and all these factors have to be considered when selecting the appropriate bioanalytical approach, be it LBA or LC-MS, for answering a particular question [63,64].

\section{Free, bound or total concentration}

Proteins can form complexes with other macromolecular binding partners in vivo: biopharmaceuticals will bind to their pharmacological target and/or to antidrug antibodies (ADAs), while many biomarkers have specific binding proteins. The unbound (free) and bound forms of a protein analyte exist in an equilibrium, which can easily shift when a sample is processed. Even simple dilution of a biological sample will theoretically lead to a shift of the equilibrium and a change in the free and bound concentrations [65]. The true free concentration of a protein in a complex biological matrix is, therefore, inherently difficult to establish. Methods that employ a binding step of the protein analyte, such as LC-MS with immunocapture, are often assumed to provide free concentrations, because only the free fraction is available for interaction and can be captured, but in reality the situation is more complicated. When the sample comes into contact with the binding material, the equilibrium between analyte and its endogenous binding partner is disturbed and the presence of an immobilized high-affinity binder may shift the equilibrium and result in the capture of a larger fraction. The magnitude of this fraction is difficult to predict, as it depends on the binding affinities of the protein analyte to its endogenous and capturing binding proteins, their concentrations and the time of incubation, but in the most extreme case it will be equal to the total concentration.

For a more correct estimation of the free protein concentration in a sample, the so-called free analyte quality control (QC) concept has been developed [66]. It is based on the addition of known amounts of the protein analyte and its endogenous binding partner to a QC sample that is originally free of both. Using mathematical models based on the binding affinity between the two partners, the final free analyte concentration in the QC sample 


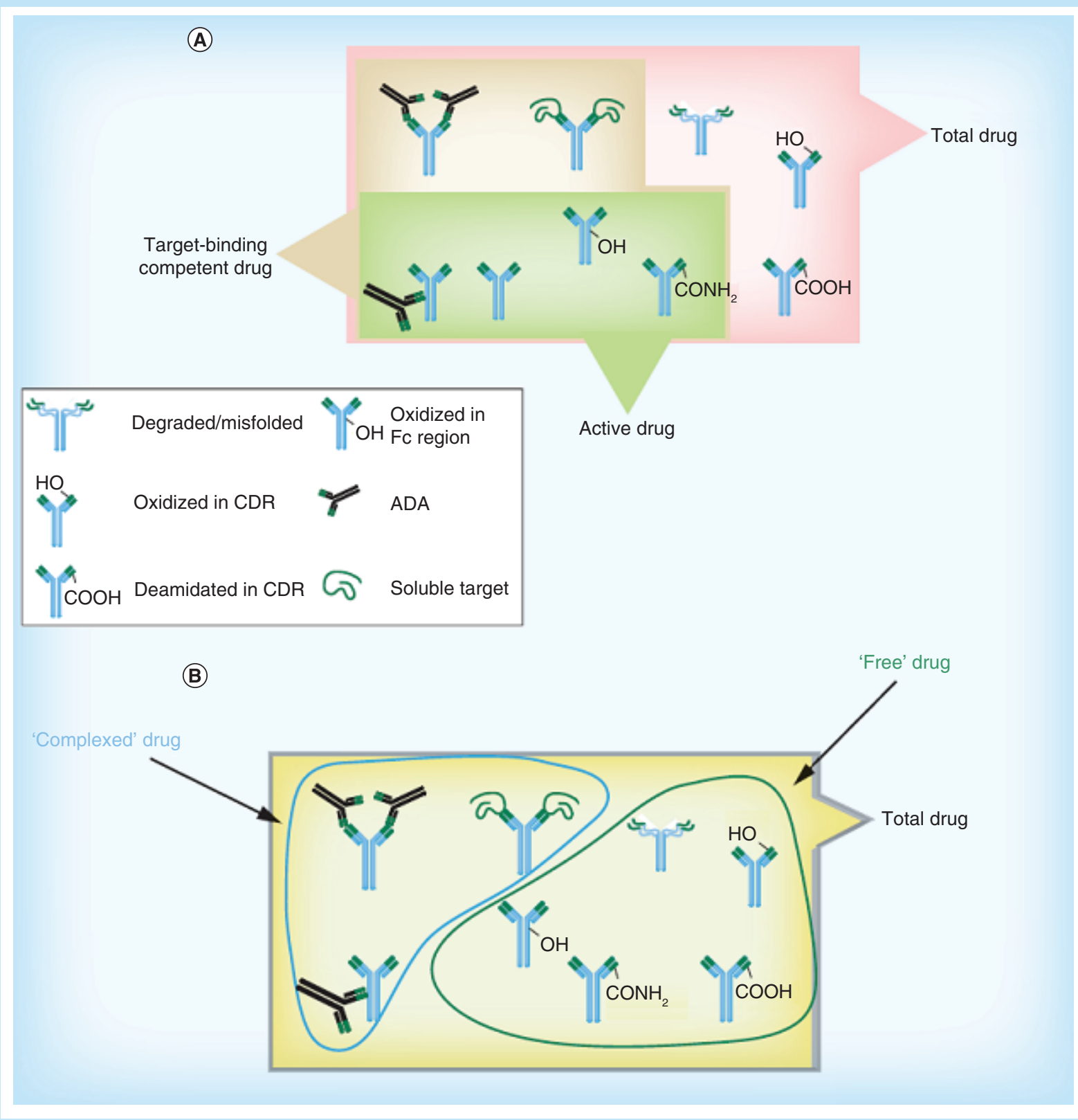

Figure 4. Occurrence of different protein forms in vivo, illustrated for a monoclonal antibody. Depicted are biotransformation (oxidation, deamidation, degradation) and binding (to ADA, soluble target), and its consequences for activity (A) and the presence or absence of drug complexes (B).

ADA: Antidrug antibody.

Reproduced from [62] ๔ Future Science Group (2015).

can be calculated. By comparison to calibrators which contain the analyte but are free from the binding protein, the free fraction is also experimentally determined. If the theoretical free fraction of the QC sample is confirmed, the assumption is that the bioanalytical method also gives correct free concentrations for any study sample. A proprietary $\mathrm{mAb}$ was quantified using affinity capture with the pharmacological target immobilized on magnetic beads, followed by trypsin digestion and LC-MS/MS analysis of a surrogate peptide. A 10-min incubation step of sample and beads provided free concentrations that agreed with the theoretical prediction, but overnight incubation resulted in a considerable overestimation, probably because the original equilibrium of analyte and its endogenous target had shifted [67]. 
Although the free concentration of a protein drug is responsible for the pharmacological effect and its determination is useful, it is practically more straightforward to determine a total concentration. Digestion of a biological sample without further protein extraction cleaves all matrix proteins into peptides and will, in principle, break all existing protein-protein interactions. Still, it is desirable to experimentally check if the digestion conditions are appropriate for quantification of the total concentration. In a report on direct digestion of trastuzumab in serum, it was observed that the addition of polyclonal ADAs resulted in a considerable negative bias of the serum concentration results [68], which suggests that the digestion conditions were insufficient to cleave the protein-ADA complex to the same extent as the free protein. The simple addition of DMSO resolved this issue, probably by making the complex more accessible to the digestion enzyme. Alternatively, an acid dissociation step will often do the job, as was demonstrated for a PEGylated therapeutic protein [13]. The addition of an equal volume of $10 \%$ formic acid to human serum before pellet digestion helped achieve correct total drug concentrations in the presence of ADAs and the soluble target.

If both free and total protein concentrations are needed, for example, to study the target engagement of a drug, complementary sample processing steps can be used. Free concentrations of the biomarker IP-10 were determined in serum by capturing this protein with an immobilized anti-IP-10 antibody that was unable to pick up the target protein when it was bound to an investigational anti-IP-10 mAb. By replacing the capturing agent by an antibody that recognizes another part of the IP-10 molecule, IP-10 was captured both in its free form and when bound to the $\mathrm{mAb}$, thus providing the total concentration [69]. A distinction between the free and ADA-bound forms of the therapeutic enzyme $\alpha$-glucosidase was made by capturing the entire IgG fraction of human plasma, including ADAs binding the protein analyte, on a protein $\mathrm{G}$ resin and removing the free fraction by washing steps [70]. After elution, the bound fraction was determined by digestion and quantification of a surrogate peptide.

\section{Biotransformation}

Despite the thorough characterization of therapeutic proteins in pharmaceutical formulations to guarantee their quality before dosing to patients, there has been relatively little attention for modifications occurring in vivo, and their impact on drug safety and efficacy after dosing. Still, biotransformation of proteins is known to take place by reactions such as oxidation, deamidation and isomerization of amino acids and truncation of peptide chains, next to release of the small-molecule drugs (payloads) from ADCs and hydrolysis of the PEG-protein bonds in PEGylated products $[71,72]$. These structural modifications may be difficult to pick up. Deamidation, for example, leads to a mass increase of just one atomic mass unit, while isomerization only results in a $3 \mathrm{D}$ rearrangement of the protein structure. Deamidation of an asparagine in the binding region of trastuzumab, with potential implications for its activity, was found by pellet digestion of patient plasma and quantification of five surrogate peptides that were chromatographically separated: one containing the undeamidated asparagine, three containing the formed aspartate, iso-aspartate and a succinimide intermediate, and one reference peptide from a stable part of the protein. All peptides were found in patient samples, thereby demonstrating extensive biotransformation [73]. Similarly, asparagine deamidation and aspartate isomerization were found in two investigational $\mathrm{mAbs}$, which were isolated from monkey serum by an antihuman IgG antibody [74]. Affinity capture of biotransformation products presents a risk, because the structural changes in the protein may lead to a reduced or even lost recognition by the capturing reagents and, thus, to considerable underestimation of the analyte concentration. Enrichment of trastuzumab from human serum by two different capture formats, based on the pharmacological target, showed substantial differences in the recovered analyte concentrations and this was speculated to be due to biotransformation in the binding region of the analyte [75].

The biotransformation of different parts of a protein can be followed by monitoring multiple surrogate peptides. Dulaglutide, a fusion protein of GLP-1 linked to the Fc domain of IgG4, was extracted from mouse plasma by an antihuman Fc antibody and digested. Two peptides were quantified, one (N-terminal) peptide from the GLP-1 part of the drug and the other from the $\mathrm{Fc}_{\mathrm{c}}$ part. While the concentrations of the $\mathrm{Fc}_{\mathrm{c}}$ part remained high after dosing, the concentrations found for the GLP-1 part decreased and correlated with the concentrations found for the intact protein, as determined with LC-HRMS. Altogether, this suggests biotransformation, and probably proteolytical cleavage, in the N-terminal part of the drug [76]. A similar approach was followed to study the in vivo fate of a protein-drug conjugate (PDC): a surrogate peptide from the unconjugated part of the protein was measured next to a peptide containing a small-molecule drug attached via a linker. After enrichment using immobilized metal affinity chromatography, the sample was digested and the two peptides were quantified as a measure for the total and conjugated forms of the PDC, respectively [77]. Biotransformation has also been investigated at the intact 
protein level by affinity capture and quantification of a fusion protein and two catabolites by LC-HRMS with deconvolution. Rapid formation of a truncated form of the dosed protein and further truncation were observed after administration to a rabbit [78].

The in vivo fate of an ADC is particularly complex, because of the release of the small-molecule payload, including or excluding its linker, and modifications to the intact ADC at the protein and/or payload structure. Therefore, a combination of techniques is required for elucidating the biotransformation of ADCs, such as analysis at the intact protein, subdomain and peptide level as well as quantification of the small-molecule drug and its possible metabolites [79,80]. The concentration of conjugated payload, in other words, all drug molecules that are still bound to an ADC after administration, can be determined by immunocapture with a payload-directed antibody, followed by release of the payload, for example, by a lysosomal enzyme, and its quantification by LC-MS/MS [81,82]. Total antibody levels have been quantified by digestion and measurement of a surrogate peptide after immunocapture with an anti-idiotypic antibody, which captures all ADCs independent of the number of drug molecules. The same approach but using immunocapture with an antipayload antibody, gives the levels of conjugated antibody, in other words, all ADCs with at least one drug [83]. More detailed information about the (remaining) number of drug molecules on an ADC can be obtained by HRMS. This so-called drug-antibody ratio (DAR) has been assessed by immunocapture of the ADC with an anti-idiotypic antibody and subsequent determination of the molecular masses of all different ADC species, from which the different DARs in a sample can be derived [84,85]. The kinetics of in vivo drug release from an ADC can depend on the way the drug is linked to the antibody. Two different ADCs with four drug molecules per antibody were studied in monkey plasma by capture with an antihuman IgG antibody and disulfide reduction, followed by LC-Q-TOF-MS analysis of the formed light (25-kDa) and heavy $(50-\mathrm{kDa})$ chains. The original DAR of four remained constant during 3 weeks for an ADC with drug attached to the protein via engineered glutamine linkers, whereas it decreased to three over the same period for an ADC containing engineered cysteine linkers [86]. By including IdeS digestion, an ADC is reduced to fragments of around $25 \mathrm{kDa}$, from which the mass, and subsequently the DAR, can also be determined [87].

\section{Isoforms}

Like biopharmaceuticals, endogenous proteins very often are not single, well-defined species, but exist in multiple, structurally different forms, called isoforms or proteoforms. Depending on the clinical question to be answered, the concentrations of one, several or all of these may be relevant. It is, therefore, important to select an appropriate bioanalytical technique that responds to all isoforms of interest. In a review paper, published in 2016, the relevance of this was indicated for a number of biomarkers [88]. The (intact) 68-amino acid protein RANTES was quantified in human plasma after immunocapture and, next to the full-length protein, several C-terminally and N-terminally truncated forms were found with potential relevance for a patient's state of health. Likewise, the levels of two truncated forms of retinol-binding protein were quantified and found to be elevated in patients with renal failure, and varying concentrations of up to nine isoforms of serum amyloid A were identified in plasma from healthy subjects and patients with Type 2 diabetes or chronic kidney disease. A proper design of the analytical method is very important in this context. Often, only one (recombinant) form of the protein is available as reference standard. If an immunocapture step is included in the workflow, the specificity of the reagent determines if all isoforms are extracted from the biological sample to the same extent. If this is not the case, incorrect concentrations of some of the isoforms would be obtained when quantified against the single recombinant standard. In addition, digestion of the protein isoforms and quantification of surrogate peptides may be a risk, in that some isoforms may only yield useful peptides that are structurally similar, so that they can no longer be separately determined. These issues were addressed in an LC-HRMS method for four isoforms of the biological toxin abrin in human plasma and other complex matrices [89]. An immunocapture step with a mixture of antibodies directed against the different isoforms was employed, in combination with quantification of 14 different surrogate peptides, some common and some isoform-specific. Because of the lack of protein standards, calibrators were used containing the surrogate peptides.

\section{Method validation}

Current regulatory guidance documents are still mainly based on the traditional bioanalytical landscape with protein quantification being performed by LBA and small-molecule quantification by LC-MS. They are not completely applicable to newer approaches that include digestion, and hybrid techniques such as immunocapture followed by LC-MS. Now that protein bioanalysis by LC-MS is entering the regulatory field, finetuning of bioanalytical best practices becomes more and more important. Validation experiments have been suggested to address new parameters 
like immunocapture efficiency and digestion efficiency, and alternative approaches for assessing matrix effect and selectivity/specificity [90]. When it comes to acceptance of results, there is ongoing debate about which criteria to apply: those traditionally used for LC-MS or those traditionally used for proteins (by LBA). Recommendations of industry experts have been published and current thinking is that, because of the complexity of the developing science of protein bioanalysis by LC-MS, there is no compelling reason to raise the standard over the currently accepted criteria for LBA [91,92].

\section{Conclusion}

Since the start of this journal 10 years ago, LC-MS has emerged as a quantitative tool for protein bioanalysis, as an alternative for or an addition to the traditional LBA-based approaches. Next to its reliable quantitative performance, the main advantage of LC-MS is related to its ability to provide structural information about a protein, or at least part of a protein, which is inherently impossible by LBA. A weaker point is its often limited detection sensitivity, but much technical progress has been made over the last decade. For many applications, sufficient sensitivity can now be obtained, in particular when LC-MS is used in combination with an (immuno)capture step. LC-MS is especially attractive for preclinical drug development, where concentrations are relatively high and the structures of the human or humanized protein drugs of interest allow a relatively simple extraction from and detection in animal matrices. In human samples, the use of LC-MS is more complicated because of the close structural similarity of analyte and endogenous matrix proteins. A risk of protein LC-MS and LBA alike, is the fact that the required method sensitivity and selectivity often dictate the choice for a particular technical approach, which may not always provide concentration results that are meaningful. The occurrence of protein isoforms and biotransformation, as well as the binding to one or more endogenous macromolecules, result in a variety of protein forms in a sample that may, may not or may only partly respond to the selected bioanalytical technique. Protein concentration results should, therefore, always be evaluated by referring to the experimental approach that was followed and the materials that were used.

\section{Future perspective}

LC-MS for quantitative protein bioanalysis is here to stay. In the near future, it will not replace but increasingly complement LBAs in drug development and patient care, because it adds value in our understanding of the in vivo fate of dosed biopharmaceuticals and endogenous biomarkers. From a technical point of view, it is to be expected that more developments will occur in the field of intact protein analysis. HRMS is increasingly being introduced in laboratories for quantitative bioanalysis and with these instruments becoming more sensitive, robust and affordable, more scientists will likely try to move from digestion of a protein and indirect quantification via a surrogate peptide to direct analysis of the intact analyte. In this respect, technical advances are needed when it comes to stationary phases for LC and it is hoped that the increased interest in intact protein analysis will stimulate the development of materials with improved separation properties for protein forms. Similarly, user-friendly software solutions for robust deconvolution of the highly complex mass spectra, in compliance with regulatory expectations, are desirable. Not less importantly, as our knowledge of protein bioanalysis increases and more and more technical possibilities become available, it can be anticipated that there will be more attention for selection of the best, or rather most appropriate, method. The usefulness of concentration results will improve if the bioanalytical approach is based on the specific scientific question to be answered, rather than using a technique that happens to be around. Finally, protein bioanalysis is a truly interdisciplinary science and much of its success is due to the collaboration of researchers with very different backgrounds. It is our hope and expectation that the bioanalytical community will continue to work together to help advance our knowledge in this fascinating field.

\section{Financial \& competing interests disclosure}

The author has no relevant affiliations or financial involvement with any organization or entity with a financial interest in or financial conflict with the subject matter or materials discussed in the manuscript. This includes employment, consultancies, honoraria, stock ownership or options, expert testimony, grants or patents received or pending, or royalties.

No writing assistance was utilized in the production of this manuscript. 
- LC-MS has become a well-established bioanalytical platform for protein quantification.

- Over the past decade, developments have focused on enhancing the technical possibilities of protein LC-MS and on improving our understanding of what a concentration result means.

- Very often, protein quantification is based on digestion of the analyte and subsequently measuring one or more surrogate peptides, but methods for intact proteins are increasingly being used.

- By properly combining extraction, digestion, separation and detection approaches, protein concentrations down to the $\mathrm{pg} / \mathrm{ml}$ level can be reliably determined.

- Conceptually, protein quantification usually is not straightforward because of the potential occurrence of many different protein forms in a sample.

- Since the technical approach that is used typically determines which protein form is quantified, it is important to have a good understanding of both the bioanalytical question and the bioanalytical method.

\section{References}

Papers of special note have been highlighted as: • of interest; $\bullet \bullet$ of considerable interest

1. Campbell JL, Le Blanc JCY. Peptide and protein drug analysis by MS: challenges and opportunities for the discovery environment. Bioanalysis 3(6), 645-657 (2011).

2. Ewles M, Goodwin L. Bioanalytical approaches to analyzing peptides and proteins by LC-MS/MS. Bioanalysis 3(12), 1379-1397 (2011).

3. Li F, Fast D, Michael S. Absolute quantitation of protein therapeutics in biological matrices by enzymatic digestion and LC-MS. Bioanalysis 3(21), 2459-2480 (2011).

4. Szapacs M, Mencken T, Williams J, Li Y. Utilizing digestion procedures in the bioanalytical laboratory. Bioanalysis 8(1), 29-36 (2016).

5. Zhang G, Sexton DJ, Faucette RR et al. 2D-LC-MS/MS to measure cleaved high-molecular weight kininogen in human plasma as a biomarker for C1-INH-HAE. Bioanalysis 9(19), 1477-1491 (2017).

6. Hansen K, Szarka S, Escoffier E et al. Glu-C, an alternative digestive enzyme for the quantitative LC-MS/MS analysis of an IgG-based antibody biotherapeutic. Bioanalysis 10(13), 997-1007 (2018).

7. Fung EN, Zambito F, Haulenbeek J et al. Targeting an acid labile aspartyl-prolyl amide bond as a viable alternative to trypsin digestion to generate a surrogate peptide for LC-MS/MS analysis. Bioanalysis 6(22), 2985-2998 (2014).

8. Ouyang Z, Furlong MT, Wu S et al. Pellet digestion: a simple and efficient sample preparation technique for LC-MS/MS quantification of large therapeutic proteins in plasma. Bioanalysis 4(10), 17-28 (2012).

- First report about the advantages of pellet digestion.

9. Yuan L, Arnold ME, Aubry A-F, Ji QC. Simple and efficient digestion of a monoclonal antibody in serum using pellet digestion: comparison with traditional digestion methods in LC-MS/MS bioanalysis. Bioanalysis 4(24), 2887-2896 (2012).

10. Shida H, Naito T, Shibata K et al. LC-MS/MS method for denosumab quantitation in human serum with rapid protein digestion using immobilized trypsin. Bioanalysis 10(18), 1501-1510 (2018).

11. Regnier FE, Kim J. Accelerating trypsin digestion: the immobilized enzyme reactor. Bioanalysis 6(19), 2685-2698 (2014).

12. Iwamoto N, Takanashi M, Umino Y et al. Application of nano-surface and molecular-orientation limited proteolysis to LC-MS bioanalysis of cetuximab. Bioanalysis 8(10), 1009-1020 (2016).

13. Gong C, Zeng J, Akinsanya B et al. Development and validation of an LC-MS/MS assay for the quantitation of a PEGylated anti CD28 domain antibody in human serum: overcoming interference from antidrug antibodies and soluble target. Bioanalysis 6(18), 2371-2383 (2014).

14. Bronsema KJ, Bischoff R, van de Merbel NC. High-sensitivity LC-MS/MS quantification of peptides and proteins in complex biological samples: the impact of enzymatic digestion and internal standard selection on method performance. Anal. Chem. 85(20), 9528-9535 (2013).

15. Wright K, Dufield D. Minimalistic sample preparation strategies for LC-MS quantification of large molecule biopharmaceuticals: a case study highlighting alpha-1 antitrypsin protein. Bioanalysis 6(13), 1813-1825 (2014).

16. Pu J, An B, Vazvaei F, Qu J. Bioanalysis. Enrichment of protein therapeutics and biomarkers for LC-MS quantification. Bioanalysis 10(13), 979-982 (2018).

17. Wilffert D, Bischoff R, van de Merbel NC. Antibody-free workflows for protein quantification by LC-MS/MS. Bioanalysis 7(6), 763-779 (2015).

- Comprehensive overview of generic protein-enrichment possibilities.

18. Wilffert D, Asselman A, Donzelli R et al. Highly sensitive antibody-free $\mu \mathrm{LC}-\mathrm{MS} / \mathrm{MS}$ quantification of rhTRAIL in serum. Bioanalysis 8(9), 881-890 (2016). 
19. Bronsema KJ, Bischoff R, Bouche M-P et al. High-sensitivity quantitation of a Nanobody ${ }^{\circledR}$ in plasma by single-cartridge multidimensional SPE and ultra-performance LC-MS/MS. Bioanalysis 7(1), 53-64 (2015).

20. Wilffert D, Donzelli R, Asselman A et al. Quantitative antibody-free LC-MS/MS analysis of sTRAIL in sputum and saliva at the sub ng/mL level. J. Chromatogr. B 1032, 205-210 (2016).

21. Shi J, Wong JM, Ma J et al. Reagent-free LC-MS/MS-based pharmacokinetic quantification of polyhistidine-tagged therapeutic proteins. Bioanalysis 9(3), 251-264 (2017).

22. Fung EN, Bryan P, Kozhich A. Techniques for quantitative LC-MS/MS analysis of protein therapeutics: advances in enzyme digestion and immunocapture. Bioanalysis 8(8), 847-856 (2016).

23. Zhao Y, Gu H, Zheng N, Zeng J. Critical considerations for immunocapture enrichment LC-MS bioanalysis of protein therapeutics and biomarkers. Bioanalysis 10(13), 987-995 (2018).

24. Vialaret J, Broutin S, Pugnier C et al. What sample preparation should be chosen for targeted MS monoclonal antibody quantification in human serum? Bioanalysis 10(10), 723-735 (2018).

25. Sleczka BG, Mehl JT, Shuster DJ et al. Quantification of human mAbs in mouse tissues using generic affinity enrichment procedures and LC-MS detection. Bioanalysis 6(13), 1795-1811 (2014).

26. Lee JW. Generic method approaches for monoclonal antibody therapeutics analysis using both ligand binding and LC-MS/MS techniques. Bioanalysis 8(1), 19-27 (2016).

27. Chen L, Liu S, Pagels S et al. Development of an ELISA-LC-MS hybrid assay for quantification of biotherapeutics. Bioanalysis 10(17), 1427-1438 (2018).

28. Wang Q, Han J, Sha C et al. Novel strategy using tryptic peptide immunoaffinity-based LC-MS/MS to quantify denosumab in monkey serum. Bioanalysis 9(19), 1451-1463 (2017).

29. Gupta V, Lassman ME, Lee AYH et al. An evaluation of an aptamer for use as an affinity ligand reagent with MS: PCSK9 as an example protein. Bioanalysis 8(15), 1557-1564 (2016).

30. Klont F, Hadderingh M, Horvatovich P et al. Affimers as an alternative to antibodies in an affinity LC-MS assay for the quantification of the soluble receptor of advanced glycation end-products (sRAGE) in human serum. J. Prot. Res. 17(8), 2892-2899 (2018).

31. Stevenson D, El-Sharif HF, Reddy SM. Selective extraction of proteins and other macromolecules from biological samples using molecular imprinted polymers. Bioanalysis 8(21), 2255-2263 (2016).

32. Yang W, Kernstock R, Simmons N, Alak A. ELISA microplate: a viable immunocapture platform over magnetic beads for immunoaffinity-LC-MS/MS quantitation of protein therapeutics? Bioanalysis 7(3), 307-318 (2015).

33. Lanshoeft C, Heudi O, Cianferani $\mathrm{S}$ et al. Quantitative analysis of hIgG1 in monkey serum by LC-MS/MS using mass spectrometric immunoassay. Bioanalysis 8(10), 1035-1049 (2016).

34. Becker JO, Hoofnagle AN. Replacing immunoassays with tryptic digestion-peptide immunoaffinity enrichment and LC-MS/MS. Bioanalysis 4(3), 281-290 (2012).

35. Sucharski FK, Meier S, Miess C et al. Development of an automated, interference-free, 2D-LC-MS/MS assay for quantification of a therapeutic mAb in human sera. Bioanalysis 10(13), 1023-1037 (2018).

36. Schultz GA, McCardle K, Neubert H. Large-scale implementation of sequential protein and peptide immunoaffinity enrichment LC/nanoLC-MS/MS for human $\beta$-nerve growth factor. Bioanalysis 8(8), 753-764 (2016).

-. Impressive methodology for routine trace-level quantification of a biomarker by pre- and postdigestion immunocapture.

37. Howard JW, Kay RG, Pleasance S, Creaser CS. UHPLC for the separation of proteins and peptides. Bioanalysis 4(24), 2971-2988 (2012).

38. Kleinnijenhuis AJ, Ingola $\mathrm{M}$, Toersche $\mathrm{JH}$ et al. Quantitative bottom up analysis of infliximab in serum using protein A purification and integrated $\mu \mathrm{LC}$-electrospray chip IonKey MS/MS technology. Bioanalysis 8(9), 891-904 (2016).

39. Lassman ME, Fernandez-Metzler C. Applications of low-flow LC-SRM for the analysis of large molecules in pharmaceutical R\&D. Bioanalysis 6(13), 1859-1867 (2014).

40. Shi T, Qian W-J. Antibody-free PRISM-SRM for multiplexed protein quantification: is this the new competition for immunoassays in bioanalysis? Bioanalysis 5(3), 267-269 (2013).

41. Sandra K, Mortier K, Jorge L et al. LC-MS/MS quantification of next-generation biotherapeutics: a case study for an IgE binding Nanobody in cynomolgus monkey plasma. Bioanalysis 6(9), 1201-1213 (2014).

42. Sandra K, Sandra P. The opportunities of 2D-LC in the analysis of monoclonal antibodies. Bioanalysis 7(22), 2843-2847 (2015).

43. Xu K, Liu L, Maia M et al. A multiplexed hybrid LC-MS/MS pharmacokinetic assay to measure two co-adminstered monoclonal antibodies in a clinical study. Bioanalysis 6(13), 1781-1794 (2014).

44. Zhu Y, D'Arienzo C, Lou Z et al. LC-MS/MS multiplexed assay for the quantitation of a therapeutic protein BMS-986089 and the target protein Myostatin. Bioanalysis 8(3), 193-204 (2016).

45. Mekhssian K, Mess J-N, Garofolo F. Application of high-resolution MS in the quantification of a therapeutic monoclonal antibody in human plasma. Bioanalysis 6(13), 1767-1779 (2014). 
46. Plumb RS, Fujimoto G, Mather J et al. Comparison of the quantification of a therapeutic protein using nominal and accurate mass MS/MS. Bioanalysis 4(5), 605-615 (2012).

47. Bults $\mathrm{P}$, Meints M, Sonesson A et al. Improving selectivity and sensitivity of protein quantitation by LC-HR-MS/MS: determination of somatropin in rat plasma. Bioanalysis 10(13), 1009-1021 (2018).

48. Kay RG, Hoard J, Stensson S. A current perspective of supercharging reagents and peptide bioanalysis. Bioanalysis 8(3), 157-161 (2016).

49. Furlong MT, Zhao S, Mylott W et al. Dual universal peptide approach to bioanalysis of human monoclonal antibody protein drug candidates in animal studies. Bioanalysis 5(11), 1363-1376 (2013).

-. Description of a universal approach to quantify human monoclonal antibodies in animal matrices by a single LC-MS method.

50. Furlong MT, Titsch C, Xu W et al. An exploratory universal LC-MS/MS assay for bioanalysis of hinge region-stabilized human IgG4 mAbs in clinical studies. Bioanalysis 6(13), 1747-1758 (2014).

51. Kaur S, Liu L, Cortes DF et al. Validation of a biotherapeutic immunoaffinity-LC-MS/MS assay in monkey serum: 'plug-and-play' across seven molecules. Bioanalysis 8(15), 1565-1577 (2016).

52. Van den Broek I, van Dongen WD. LC-MS-based quantification of intact proteins: perspective for clinical and bioanalytical applications. Bioanalysis 7(15), 1943-1958 (2015).

53. Lanshoeft C, Cianferani S, Heudi O. Generic hybrid ligand binding assay liquid chromatography high-resolution mass spectrometry-based workflow for multiplexed human immunoglobulin G1 quantification at the intact protein level: application to preclinical pharmacokinetic studies. Anal. Chem. 89(4), 2628-2635 (2017).

54. Ramagiri S, Garofolo F. Large molecule bioanalysis using Q-TOF without predigestion and its data processing challenges. Bioanalysis 4(5), 529-540 (2012).

55. Jian W, Kang L, Burton L, Weng N. A workflow for absolute quantitation of large therapeutic proteins in biological samples at intact level using LC-HRMS. Bioanalysis 8(16), 1679-1691 (2016).

-. First report of the possibility to quantify an intact monoclonal antibody by LC-MS.

56. Qiu X, Kang L, Case M et al. Quantitation of intact monoclonal antibody in biological samples: comparison of different data processing strategies. Bioanalysis 10(13), 1055-1067 (2018).

57. Jin W, Burton L, Moore I. LC-HRMS quantitation of intact antibody drug conjugate trastuzumab emtansine from rat plasma. Bioanalysis 10(11), 851-862 (2018).

58. Kellie JF, Kehler JR, Karlinsey MZ, Summerfield SG. Toward best practices in data processing and analysis for intact biotherapeutics by MS in quantitative bioanalysis. Bioanalysis 9(23), 1883-1893 (2017).

59. Zhang L, Vasicek LA, Hsieh SC et al. Top-down LC-MS quantitation of intact denatured and native monoclonal antibodies in biological samples. Bioanalysis 10(13), 1039-1054 (2018).

- Insightful report on the possibility for quantitative bioanalysis of large proteins in their native form.

60. Kellie JF, Kehler JR, Mencken T et al. A whole-molecule immunocapture LC-MS approach for the in vivo quantitation of biotherapeutics. Bioanalysis 8(20), 2103-2114 (2016).

61. Van de Merbel NC, Bronsema KJ, Nemansky M. Protein quantification using LC-MS: can it make a difference? Bioanalysis 4(17), 2113-2116 (2012).

62. Heinrich J, Staack RF, Stubenrauch K-G, Papadimitriou A. Proposal for a harmonized descriptive analyte nomenclature for quantitative large-molecule bioanalysis. Bioanalysis 7(24), 3057-3062 (2015).

63. Zhang YJ, Olah TV, Zeng J. The integration of ligand binding and LC-MS-based assays into bioanalytical strategies for protein analysis. Bioanalysis 6(13), 1827-1841 (2014).

64. Zhang YJ, Luo L, Desai DD. Overview on biotherapeutic proteins: impact on bioanalysis. Bioanalysis 8(1), 1-9 (2016).

65. White JT, Bonilla LE. Free and total biotherapeutic evaluation in chromatographic assays: interference from targets and immunogenicity. Bioanalysis 4(19), 2401-2411 (2012).

-• Excellent overview of the analytical consequences of protein-protein interactions.

66. Staack RF, Jordan G, Dahl G, Heinrich J. Free analyte concept: a novel approach to prove correct quantification of free therapeutic protein drug/biomarker concentrations. Bioanalysis 6(4), 485-496 (2014).

67. Jordan G, Onami I, Heinrich J, Staack RF. Evaluation of the potential use of hybrid LC-MS/MS for active drug quantification applying the 'free analyte QC concept'. Bioanalysis 9(21), 1705-1717 (2017).

-. Convincing description of the possibilities to establish the free concentration of a protein by LC-MS.

68. Ewles M, Mannu R, Fox C et al. LC-MS/MS strategies for therapeutic antibodies and investigation into the quantitative impact of anti-drug antibodies. Bioanalysis 8(24), 2565-2579 (2016).

69. Zhang H, Gu H, Shipkova P et al. Immunoaffinity LC-MS/MS for quantitative determination of a free and total protein target as a target engagement biomarker. Bioanalysis 9(20), 1573-1588 (2017). 
70. Bronsema KJ, Bischoff R, Pijnappel WWMP et al. Absolute quantification of the total and antidrug antibody-bound concentrations of recombinant human $\alpha$-glucosidase in human plasma using protein G extraction and LC-MS/MS. Anal. Chem. 87(8), 4394-4401 (2015).

71. Hamuro LL, Kishnani NS. Metabolism of biologics: biotherapeutic proteins. Bioanalysis 4(2), 189-195 (2012).

72. Kellie JF, Karlinsey MZ. Review of approaches and examples for monitoring biotransformation in protein and peptide therapeutics by MS. Bioanalysis 10(22), 1877-1890 (2018).

- Good overview of protein biotransformation and its bioanalytical consequences.

73. Bults $\mathrm{P}$, Bischoff R, Bakker $\mathrm{H}$ et al. LC-MS/MS-based monitoring of in vivo protein biotransformation: quantitative determination of tratstuzumab and its deamidation products in human plasma. Anal. Chem. 88(3), 1871-1877 (2016).

74. Mehl JT, Sleczka BG, Ciccimaro F et al. Quantification of in vivo site-specific Asp isomerization and Asn deamidation of $\mathrm{mAbs}$ in animal serum using IP-LC-MS. Bioanalysis 8(15), 1611-1622 (2016).

75. Liu L, Xu K, Li J et al. Optimizing hybrid LC-MS/MS binding conditions is critical: impact of biotransformation on quantification of trastuzumab. Bioanalysis 10(22), 1819-1831 (2018).

76. Kang L, Camacho RC, Li W et al. Simultaneous catabolite identification and quantitation of large therapeutic protein at the intact level by immunoaffinity capture liquid chromatography-high-resolution mass spectrometry. Anal. Chem. 89(11), 6065-6075 (2017).

77. Shi C, Goldberg S, Lin T et al. Bioanalytical workflow for novel scaffold protein-drug conjugates: quantitation of total centyrin protein, conjugated centyrin and free payload for centyrin-drug conjugate in plasma and tissue samples using liquid chromatography-tandem mass spectrometry. Bioanalysis 10(20), 1651-1665 (2018).

78. Zell M, Husser C, Staack RF et al. In vivo biotransformation of the fusion protein tetranectin-apolipoprotein A1 analyzed by ligand-binding mass spectrometry combined with quantitation by ELISA. Anal. Chem. 88(23), 11670-11677 (2016).

79. Saad OM, Shen B-Q, Xu K et al. Bioanalytical approaches for characterizing catabolism of antibody-drug conjugates. Bioanalysis 7(13), 1583-1604 (2015).

- Perspective on the bioanalysis of antibody-drug conjugates and their biotransformation products.

80. Tumey LN, Rago B, Han X. In vivo biotransformation of antibody-drug conjugates. Bioanalysis 7(13), 1649-1664 (2015).

81. Myler H, Rangan VS, Wang J et al. An integrated multiplatform bioanalytical strategy for antibody-drug conjugates: a novel case study. Bioanalysis 7(13), 1569-1582 (2015).

82. Sanderson RJ, Nicholas ND, Baker Lee C et al. Antibody-conjugated drug assay for protease-cleavable antibody-drug conjugates. Bioanalysis 8(1), 55-63 (2016).

83. Wang J, Gu H, Liu A et al. Antibody-drug conjugate bioanalysis using LB-LC-MS/MS hybrid assays: strategies, methodology and correlation to ligand-binding assays. Bioanalysis 8(13), 1383-1401 (2016).

84. Kaur S, Xu K, Saad OM et al. Bioanalytical assay strategies for the development of antibody-drug conjugate biotherapeutics. Bioanalysis 5(2), 201-226 (2013).

85. Xu K, Liu L, Dere R et al. Characterization of the drug-to-antibody ratio distribution for antibody-drug conjugates in plasma/serum. Bioanalysis 5(9), 1057-1071 (2013).

86. Grafmuller L, Wei C, Ramanathan R et al. Unconjugated payload quantification and DAR characterization of antibody-drug conjugates using high-resolution MS. Bioanalysis 8(16), 1663-1678 (2016).

87. Davis JA, Kagan M, Read J et al. Immunoprecipitation middle-up LC-MS for in vivo drug-to-antibody ratio determination for antibody-drug conjugates. Bioanalysis 9(20), 1535-1549 (2017).

88. Trenchevska O, Nelson RW, Nedelkov D. Mass spectrometric immunoassays for discovery, screening and quantification of clinically relevant proteoforms. Bioanalysis 8(15), 1623-1633 (2016).

89. Hansbauer E-M, Worbs S, Volland $\mathrm{H}$ et al. Rapid detection of abrin toxin and its isoforms in complex matrices by immuno-extraction and quantitative high resolution targeted mass spectrometry. Anal. Chem. 89(21), 11719-11727 (2017).

90. Duggan J, Ren B, Mao Y et al. LC-MS quantification of protein drugs: validating protein LC-MS methods with predigestion immunocapture. Bioanalysis 8(18), 1951-1964 (2016).

91. Knutsson M, Schmidt R, Timmerman P. LC-MS/MS of large molecules in a regulated environment - which acceptance criteria to apply? Bioanalysis 5(18), 2211-2214 (2013).

92. Duggan JX, Vazvaei F, Jenkins R. Bioanalytical method validation considerations for LC-MS/MS assays of therapeutic proteins. Bioanalysis 7(11), 1389-1395 (2015). 